\title{
Non-axisymmetric oscillations of thin twisted magnetic tubes
}

\author{
Michael S. Ruderman \\ Solar Physics and Space Plasma Research Centre ( $\left.\mathrm{SP}^{2} \mathrm{RC}\right)$, Department of Applied \\ Mathematics, University of Sheffield, Hicks Building, Hounsfield Road, Sheffield, S3 7RH, UK \\ email: M.S.Ruderman@sheffield.ac.uk
}

\begin{abstract}
In this paper we study non-axisymmetric oscillations of thin twisted magnetic tubes taking the density variation along the tube into account. We use the approximation of the zero-beta plasma. The magnetic field outside the tube is straight and homogeneous, however it is twisted inside the tube. We assume that the azimuthal component of the magnetic field is proportional to the distance from the tube axis, and that the tube is only weakly twisted, i.e. the ratio of the azimuthal and axial components of the magnetic field is small. Using the asymptotic analysis we show that the eigenmodes and eigenfrequencies of the kink and fluting oscillations are described by a classical Sturm-Liouville problem for a second order ordinary differential equation. The main result is that the twist does not affect the kink mode.
\end{abstract}

\section{Introduction}

Non-axisymmetric waves in magnetic flux tubes can be divided into kink waves and fluting waves. Fast kink waves were first studied theoretically by Ryutov \& Ryutova (1976), who obtained the expression for the phase speed of these waves. After that kink waves in magnetic tubes were studied by many authors (see, e.g., Spruit (1981), Spruit (1982); Edwin \& Roberts (1983), and references therein).

Since transverse oscillations of coronal loops were first observed by TRACE (Aschwanden et al. 1999; Nakariakov et al. 1999), and subsequently interpreted as fast kink oscillations of magnetic flux tubes by Nakariakov et al. (1999), the theory of non-axisymmetric oscillations of magnetic tubes remains among the hot topics in solar physics. For the latest review on observations of coronal loop oscillations see e.g. Banerjee el al. (2007). The most attention was given to kink oscillations. In early theoretical studies only straight homogeneous tubes with circular cross-sections were considered. In recent years the theory was extended in different directions.

However, one important effect that has not been studied extensively yet in the context of oscillations. This effect is the magnetic twist. Twisted magnetic tubes have been investigated for many years, but mainly either in the context of tube stability, or in relation to the MHD wave resonant absorption. To our knowledge the only early papers where the effect of the magnetic twist on the waves propagation was studied are those by Bogdan (1984) and Bennett et al. (1999). These authors studied the wave propagation in twisted magnetic tubes in incompressible plasmas.

Recently the wave propagation in twisted magnetic tubes started to receive an enhanced attention. Erdélyi \& Fedun (2006) considered the sausage wave propagation in magnetic tubes in incompressible plasmas with the magnetic field twisted both inside and outside the tube. Erdélyi \& Carter (2006), Carter \& Erdélyi (2007) and Carter \& Erdélyi (2008) investigated the wave propagation in a tube that consists of a core with 
straight magnetic field and an annulus with twisted magnetic field. Erdélyi \& Fedun (2007) studied the compressibility effect on the wave propagation in twisted magnetic flux tubes. However, in all these papers propagating waves were considered. To our knowledge, standing non-axisymmetric oscillations of magnetic flux tubes with fixed ends has not been studied yet.

In this paper we study the eigenmodes and eigenfrequencies of non-axisymmetric oscillations of thin magnetic tubes taking two effects into account simultaneously: magnetic twist and the density variation along the tube axis. The paper is organized as follows. In the next section we formulate the problem and write the linearized system of governing equations and boundary conditions in terms of the plasma displacement and magnetic pressure perturbation. In Section 3 we derive the eigenvalue problem determining the eigenfrequencies and eigenfunctions of the twisted tube oscillations. In Section 4 we study the eigenmodes of non-axisymmetric tube oscillations. Section 5 contains the summary of the obtained results and our conclusions.

\section{Formulation}

We consider a twisted magnetic tube of radius $a$ in a cold plasma. We introduce cylindrical coordinates $r, \varphi, z$ with the $z$-axis coinciding with the tube axis. The tube length is $L$, and the tube ends are fixed at $z=0$ and $z=L$. This corresponds to the condition that the magnetic field lines are frozen in the dense photospheric plasma. The density, $\rho$, depends on $z$ and has a jump at the tube boundary, so that

$$
\rho= \begin{cases}\rho_{i}(z), & r<a, \\ \rho_{e}(z), & r>a .\end{cases}
$$

We assume that $\rho_{e}(z)<\rho_{i}(z)$, otherwise $\rho_{i}(z)$ and $\rho_{e}(z)$ are arbitrary. In what follows the subscripts ' $e$ ' and ' $i$ ' refer to quantities ourside and inside the tube respectively.

We assume that the equilibrium magnetic field is straight and have constant magnitude $B_{0}$ outside the tube, i.e. for $r>a$. Inside the tube it is twisted, i.e. it has $z$ and $\varphi$ components, and it only depends on $r$. We choose the simplest possible dependence of the asimuthal component of the magnetic field on $r, B_{\varphi i}=A r$. The magnetic field has to satisfy the equailibrium condition inside the tube,

$$
\frac{d\left(B_{\varphi i}^{2}+B_{z i}^{2}\right)}{d r}=-\frac{2 B_{\varphi i}^{2}}{r},
$$

and at the tube boundary,

$$
B_{\varphi i}^{2}+B_{z i}^{2}=B_{e}^{2}
$$

It follows from (2.2) and (2.3) that the $z$-component of the magnetic field inside the tube is given by

$$
B_{z i}^{2}=B_{0}^{2}+2 A^{2}\left(a^{2}-r^{2}\right) .
$$

The equilibrium configuration is shown in Fig. 1.

The plasma motion is described by the system of linearized ideal MHD equations for a cold plasma. Since the equilibrium quantities are independent of $\varphi$, we Fourier-analyze the perturbations of all quantities, and take them proportional to $e^{i m \varphi}$, where $m$ is an integer, $m \neq 0$. In addition, we are looking for eigenmodes, so that we take perturbations of all variables proportional to $e^{-i \omega t}$. Then the system of linearized ideal MHD equations can be written in terms of the plasma displacement, $\boldsymbol{\xi}=\left(\xi_{r}, \xi_{\varphi}, \xi_{z}\right)$, and the perturbation 


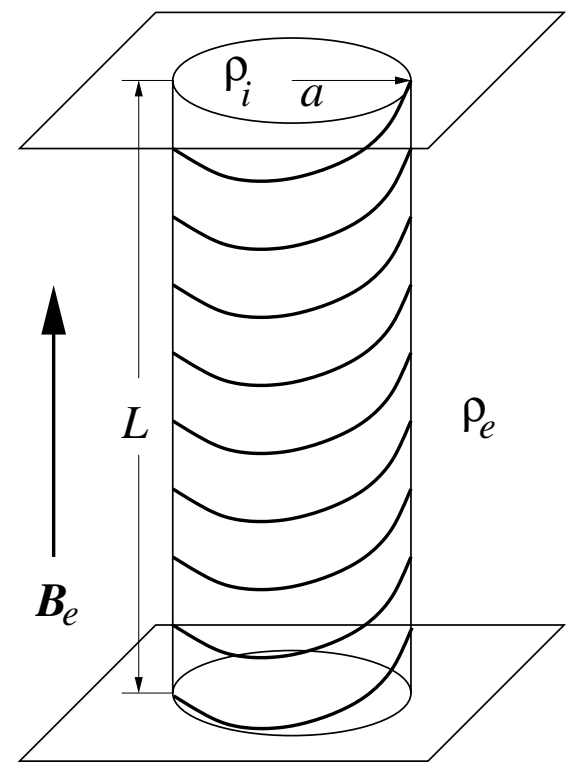

Figure 1. Twisted magnetic tube.

of the magnetic pressure, $P$, as (Ruderman (2007))

$$
\begin{gathered}
\left(\rho \omega^{2}+\frac{B^{2}}{\mu_{0}} \mathcal{D}_{\|}^{2}\right) \xi_{r}+\frac{2 A^{2}}{\mu_{0}} \frac{\partial\left(r \xi_{r}\right)}{\partial r}=\frac{\partial P}{\partial r}+\frac{2 A B}{\mu_{0}} \frac{\partial \xi_{\perp}}{\partial z}, \\
\left(\rho \omega^{2}+\frac{B^{2}}{\mu_{0}} \mathcal{D}_{\|}^{2}\right) \xi_{\perp}=\mathcal{D}_{\perp} P-\frac{2 A B_{z}}{\mu_{0}} \mathcal{D}_{\|} \xi_{r} \\
P=-\frac{B^{2}}{r \mu_{0}} \frac{\partial\left(r \xi_{r}\right)}{\partial r}+\frac{2 A^{2} r}{\mu_{0}} \xi_{r}-\frac{B^{2}}{\mu_{0}} \mathcal{D}_{\perp} \xi_{\perp}
\end{gathered}
$$

Here

$$
\xi_{\perp}=\left(B_{z} \xi_{\varphi}-B_{\varphi} \xi_{z}\right) / B, \quad P=\boldsymbol{B} \cdot \boldsymbol{b},
$$

where $\boldsymbol{B}$ is the equilibrium magnetic field, and $\boldsymbol{b}$ is the magnetic field perturbation. Note that $\xi_{\|}=\left(B_{\varphi} \xi_{\varphi}+B_{z} \xi_{z}\right) / B$ is zero in the cold plasma approximation. Operators $\mathcal{D}_{\perp}$ and $\mathcal{D}_{\|}$are given by

$$
\mathcal{D}_{\perp}=-\frac{A r}{B} \frac{\partial}{\partial z}+\frac{i m B_{z}}{r B}, \quad \mathcal{D}_{\|}=\frac{B_{z}}{B} \frac{\partial}{\partial z}+\frac{i m A}{B} .
$$

The system of equations (2.5)-(2.7) is written for motions inside the tube with the subscript ' $i$ ' omitted. To obtain the system of equation for motions outside the tube we only need to take $A=0$ in $(2.5)-(2.7)$.

The perturbations have to satisfy the kinematic and dynamic boundary conditions at $r=a$. These conditions can be written as (see Bennett 1999 or Erdélyi \& Fedun 2006)

$$
\begin{gathered}
\xi_{r i}=\xi_{r e}, \\
P_{i}-\frac{a A^{2}}{\mu_{0}} \xi_{r i}=P_{e} .
\end{gathered}
$$

In additions, all perturbations are assumed to decay as $r \rightarrow \infty$. 
The system of equations (2.5)-(2.7) with the boundary conditions (2.10) and (2.11) will be used in the next section to study the eigenmodes of the tube oscillations.

\section{Eigenvalue problem for tube oscillations}

In this section we derive the governing equation for non-axisymmetric oscillations of a thin magnetic tube with twisted magnetic field. The assumption that the tube is thin implies that $\epsilon=a / L \ll 1$. We also assume that the magnetic field inside the tube is only weakly twisted, so that $a A / B_{0}=\mathcal{O}(\epsilon)$ and introduce $\widetilde{A}=\epsilon^{-1} A$. Then it follows that $B_{z i}=B_{0}+\mathcal{O}\left(\epsilon^{2}\right)$. We also assume that $\omega \sim B_{0} / L \sqrt{\mu_{0} \rho_{i}}$ and introduce the scaled frequency $\Omega=\epsilon^{-1} \omega$. Finally, we introduce the stretching variable $\zeta=\epsilon z$.

Now we look for the solution to equations (2.5)-(2.7) in the form of series expansions with respect to $\epsilon$. Here we give only the main results. The details of calculations can be found in Ruderman (2007). In the lowest order approximation we obtain the equation for $\xi_{r}$ and the expression for $P$ in terms of $\xi_{r}$,

$$
\begin{gathered}
\left(\Omega^{2}+\frac{B_{0}^{2}}{\mu_{0} \rho_{i}} \widetilde{\mathcal{D}}_{\|}^{2}\right)\left(\frac{\partial}{\partial r} r \frac{\partial\left(r \xi_{r}\right)}{\partial r}-m^{2} \xi_{r}\right)=0, \\
P=\epsilon^{2} \frac{r}{m^{2}}\left(\rho_{i} \Omega^{2}+\frac{B_{0}^{2}}{\mu_{0}} \widetilde{\mathcal{D}}_{\|}^{2}\right) \frac{\partial\left(r \xi_{r}\right)}{\partial r}-\epsilon^{2} \frac{2 i B_{0} \widetilde{A} r}{m \mu_{0}} \widetilde{\mathcal{D}}_{\|} \xi_{r}+\mathcal{O}\left(\epsilon^{4}\right),
\end{gathered}
$$

where

$$
\widetilde{\mathcal{D}}_{\|}=\frac{\partial}{\partial \zeta}+\frac{i m \widetilde{A}}{B_{i}}
$$

The first factor equal to zero corresponds to Alfvén waves inside the tube. In what follows we eliminate Alfvén waves from the analysis, and assume that $\Omega^{2}$ is not close to any eigenvalue of the operator determined by the differential expression $-\left(B_{0}^{2} / \mu_{0} \rho_{i}\right) \widetilde{\mathcal{D}}_{\|}^{2}$ and the zero boundary conditions at $\zeta=0, \epsilon L$. More precisely, we assume that the difference between any such eigenvalue and $\Omega^{2}$ is of the order of unity. Then it follows that equation (3.1) reduces to

$$
\frac{\partial}{\partial r} r \frac{\partial\left(r \xi_{r}\right)}{\partial r}-m^{2} \xi_{r}=0
$$

The solution of this equation regular at $r=0$ is very simple,

$$
\xi_{r}=r^{|m|-1} \eta(\zeta)
$$

where $\eta(\zeta)$ is an arbitrary function satisfying $\eta(0)=\eta(\epsilon L)=0$. Using (3.5) we easily obtain

$$
P=\epsilon^{2} r^{|m|}\left[\frac{B_{0}^{2}}{\mu_{0}|m|} \frac{d^{2} \eta}{d \zeta^{2}}+\frac{2 i \widetilde{A} B_{0}(|m|-1)}{m \mu_{0}} \frac{d \eta}{d \zeta}+\frac{\widetilde{A}^{2}(2-|m|)}{\mu_{0}} \eta+\frac{\rho_{i} \Omega^{2}}{|m|} \eta\right]+\mathcal{O}\left(\epsilon^{4}\right) .
$$

Outside the tube, the system of equations (2.5)-(2.7) is reduced to the system of equations for $\xi_{r}$ and $P$,

$$
\begin{gathered}
\frac{\partial^{2} \xi_{r}}{\partial \zeta^{2}}+\frac{\mu_{0} \rho_{e} \Omega^{2}}{B_{0}^{2}} \xi_{r}=\frac{\epsilon^{-1} \mu_{0}}{B_{0}^{2}} \frac{\partial P}{\partial R}, \\
\frac{\partial^{2} P}{\partial R^{2}}+\frac{1}{R} \frac{\partial P}{\partial R}+\frac{\partial^{2} P}{\partial \zeta^{2}}+\left(\frac{\mu_{0} \rho_{e} \Omega^{2}}{B_{0}^{2}}-\frac{m^{2}}{R^{2}}\right) P=0,
\end{gathered}
$$

where $R=\epsilon r$ is the stretching variable in the radial direction. Equation (3.8) can be solved by means of variable separation. For what follows we need only to know the 
solution near the tube boundary, where $R=\mathcal{O}(\epsilon)$. For these values of $R$ the solution to (3.8) is given by the asymptotic expression

$$
P_{e}=f(\zeta)\left(\frac{\epsilon a}{R}\right)^{|m|}
$$

It follows from the boundary condition (2.10) and equation (2.7) that function $f$ satisfies $f(0)=f(\epsilon L)=0$. Otherwise it is arbitrary.

Now we match the solutions inside and outside the tube using equation (3.7), and the boundary conditions (2.10) and (2.11). It follows from (2.10) and (3.5) that $\xi_{r e}=$ $a^{|m|-1} \eta(\zeta)$. Substituting this expression in (3.7) we obtain

$$
f(\zeta)=-\epsilon^{2} a^{|m|} \frac{B_{0}^{2}}{\mu_{0}|m|}\left(\frac{d^{2} \eta}{d \zeta^{2}}+\frac{\mu_{0} \rho_{e} \Omega^{2}}{B_{0}^{2}} \eta\right) .
$$

Now we substitute $P_{e}$ with $f(\zeta)$ given by (3.10) in (2.11), use (3.5) and (3.6), and return to the original variables. As a result, after some algebra, we arrive at the following SturmLiouville problem determining the eigenfunction $\eta$ and the eigenfrequency $\omega$ of the tube oscillation:

$$
\begin{gathered}
\frac{d^{2} \eta}{d z^{2}}+\frac{i A[m-\operatorname{sgn}(m)]}{B_{0}} \frac{d \eta}{d z}+\left(\frac{\omega^{2}}{C_{k}^{2}}-\frac{A^{2}|m|(|m|-1)}{2 B_{0}^{2}}\right) \eta=0, \\
\eta=0 \quad \text { at } \quad z=0, L
\end{gathered}
$$

where

$$
C_{k}^{2}(z)=\frac{2 B^{2}}{\mu_{0}\left[\rho_{i}(z)+\rho_{e}(z)\right]}
$$

When $A=0$, equation (3.11) coincides with equation (21) in Dymova \& Ruderman (2005) for an untwisted tube. The substitution

$$
\eta=h \exp \left(-\frac{i z A[m-\operatorname{sgn}(m)]}{2 B_{0}}\right)
$$

reduces (3.11) to the standard Sturm-Liouville problem,

$$
\frac{d^{2} h}{d z^{2}}+\left(\frac{\omega^{2}}{C_{k}^{2}}-\frac{A^{2}\left(m^{2}-1\right)}{4 B_{0}^{2}}\right) h=0, \quad h=0 \quad \text { at } \quad z=0, L .
$$

This problem determines all eigenfrequencies and corresponding eigenmodes of the tube oscillation for any $m \neq 0$.

\section{Eigenmodes of tube oscillations}

The eigenvalue problem (3.14) is self-adjoint, so that all its eigenvalues are real. Obviously, we can assume that all eigenfunctions are also real. Multiplying (3.14) by $h$ and using the integration by parts we obtain

$$
\omega^{2} \int_{0}^{L} \frac{h^{2}}{C_{k}^{2}} d z=\int_{0}^{L}\left(\frac{d h}{d z}\right)^{2} d z+\frac{A^{2}\left(m^{2}-1\right)}{4 B_{0}^{2}} \int_{0}^{L} h^{2} d z .
$$

This equation shows that all eigenvalues of the Sturm-Liouville problem (3.14) are positive, so that there are no unstable modes described by (3.14).

Now we note that equation (3.14) is independent of the sign of $m$. Hence, there is only one standing mode for each $|m|>0$, and we can take $m>0$ in what follows. When $m=1$, which corresponds to the kink mode, equation (3.14) coinsides with with equation 
(21) in Dymova \& Ruderman (2005) describing kink oscillations of an untwisted tube. Hence, we obtained a very important result that weak magnetic twist does not affect the kink oscillations. However. it does affect the fluting modes. For a non-stratified tube the fundamental frequencies of non-axisymmetric modes are given by

$$
\omega_{m f}^{2}=C_{k}^{2}\left(\frac{\pi^{2}}{L^{2}}+\frac{A^{2}\left(m^{2}-1\right)}{4 B_{0}^{2}}\right) .
$$

We see that the magnetic twist increases the frequency of a fluting mode.

\section{Summary and conclusions}

In this paper we have studied non-axisymmetric oscillations of a thin twisted magnetic tube with fixed ends in a zero-beta plasma. We have taken the density stratification into account and allowed the arbitrary variation of densities inside and outside the tube in the longitudinal direction. The only condition that we imposed was that the density inside the tube is larger than that outside. We have taken the magnetic field outside the tube straight and homogeneous, while inside the tube the magnetic field has the axial and azimuthal components, the latter being proportional to the radial coordinate $r$. Then we have made a very important assumption that the twist is weak, i.e. the azimuthal component of the magnetic field inside the tube is much smaller than the axial component. More precisely, we assumed that the ratio of these two components is of the order of the ratio of the tube radius to its length, $\epsilon=a / L \ll 1$.

Using the asymptotic expansions with $\epsilon$ as a small parameter, we have shown that the eigenmodes and eigenfrequencies of the tube oscillation with perturbations of all quantities proportional to $\exp (\operatorname{im} \varphi)(m \neq 0)$ are determined by the Sturm-Liouville problem (3.14), which is a generalization of the result obtained by Dymova \& Ruderman (2005) for a straight magnetic tube.

The most important result obtained in this paper is that the weak twist does not affect the kink oscillations $(m=1)$. In this respect it is interesting to note that a similar result was obtained by Bennett et al. (1999), Carter \& Erdélyi (2008) for kink waves propagating in magnetic tubes in incompressible plasmas.

We showed that all modes described by the Sturm-Liouville problem (3.14) are stable. However, it is very important to note that this Sturm-Liouville problem describes by far not all modes that can be supported by a thin twisted magnetic tube. To show this we first recall the results concerning straight magnetic tubes. In that case, for each $m>0$, in addition to the mode described be (3.14) with $A=0$, there are infinitely many other modes. The characteristic property of the modes described by (3.14) with $A=0$ is that the plasma displacement in the radial direction takes its maximum value at the tube boundary. In contrast, for modes not described by (3.14), the plasma displacement at the tube boundary is very small, and it tends to zero as $\epsilon \rightarrow 0$. In accordance with the classification introduced by Roberts (1981), both the modes described and not described by (3.14) are body waves in the zero-beta plasma approximation. To distinguish the kink mode described by (3.14) from those not described by (3.14), Ruderman \& Roberts (2002) suggested to call it "global kink mode," retaining the name "body kink modes" for all other kink modes. We generalize this convention for fluting modes, and call fluting modes described by (3.14) "global fluting modes," retaining the name "body fluting modes" for all other fluting modes.

In the case of a thin straight tube, body kink and fluting modes are all leaky modes and, in addition, their phase speeds are tending to infinity as $\epsilon \rightarrow 0$. Hence, the assumption that $\omega \sim B_{0} / L \sqrt{\mu_{0} \rho_{i}}$ effectively eliminates the body modes from the analysis. However, 
the situation is completely different when the tube is twisted. Now the body modes are not necessarily leaky, and their phase speeds can be small even in the thin tube approximation. A preliminary analysis show that body kink and fluting modes with frequencies close to $\pi n B_{0} /\left(L \sqrt{\mu_{0} \rho_{i}}\right)$, where $n$ is a positive integer, can exist even in the approximation of incompressible plasma in an unstratified tube (e.g., Erdélyi \& Carter (2006)). To eliminate the body modes from the analysis we had to make an additional assumption that the squared eigenfrequency of the tube oscillation is not close to any of the eigenvalues of the Sturm-Liouville problem

$$
\frac{B_{0}^{2}}{\mu_{0} \rho_{i}}\left(\frac{d^{2} f}{d z^{2}}+\frac{2 i m A}{B_{0}} \frac{d f}{d z}-\frac{m^{2} A^{2}}{B_{0}^{2}} f\right)=-\sigma f, \quad f=0 \text { at } z=0, L .
$$

This eigenvalue problem describes standing Alfvén waves in the tube.

An important problem is the stability of a twisted tube. For an infinite tube this problem was partially studied by Dungey \& Loughhead (1954), Bennett et al. (1999) and Carter \& Erdélyi (2008) in the approximation of an incompressible plasma. In particular, it follows from the results obtained by these authors that, in the long wavelength approximation, the first travelling body wave (the wave that has no nodes in the radial direction inside the tube) with $m=-1$ becomes unstable when $2 \pi A / k_{z} B_{0}>1+\mathcal{O}(\epsilon)$ (see Fig. 7 in Bennett et al. 1999). The line-tying condition at the tube ends should stabilize the tube. The study of stability of a twisted tube with fixed ends is a problem for future investigation. However the conjecture that the stability criterion for such a loop has the form $L A / B_{0}<q+\mathcal{O}(\epsilon)$ with $q$ being a positive constant (unknown at present) looks quite viable. In Section 3 we assumed that $A=\epsilon \widetilde{A}$ with $a A / B_{0}=\mathcal{O}(\epsilon)$. If our conjecture about the stability criterion is correct, then a more accurate assumption should be $a A / B_{0}<q \epsilon$.

\section{References}

Aschwanden, M. J., Fletcher, L., Schrijver, C. J., \& Title, A. M. 1999, Astrophys. J. 520, 880 Banerjee, D., Erdélyi, R., Oliver, R. \& O'Shea, E. 2007, Solar Phys., 246, 3

Bennett, K., Roberts, B., \& Narain, U. 1999, Solar Phys., 185, 41

Bogdan, T. J. 1984, ApJ, 282, 769

Carter, B. K. \& Erdélyi, R. 2007, A\&A A, 475, 323

Carter, B. K. \& Erdélyi, R. 2008, A\&A A, 481, 239

Dungey, J. W. \& Loughhead, R. E. 1954, Austr. J. Phys., 7, 5

Dymova, M. \& Ruderman, M. S.: 2005, Solar Phys., 229, 79

Edwin, P. M. \& Roberts, B. 1983, Solar Phys., 88, 179

Erdélyi, R. \& Carter, B. K. 2006, A\& A, 455, 361

Erdélyi, R. \& Fedun, V. 2006, Solar Phys., 238, 41

Erdélyi, R. \& Fedun, V. 2007, Solar Phys., 246, 101

Nakariakov, V., Ofman, L., DeLuca, E. E., Roberts, B. \& Davila, J. M. 1999, Science, 285, 862

Roberts, B. 1981, Solar Phys., 69, 27

Ruderman, M. S. 2007, Solar Phys., 246, 119

Ruderman, M. S. \& Roberts, B. 2002, ApJ, 577, 475.

Ryutov, D. D. \& Ryutova, M. P. 1976, Sov. Phys.-JETP, 43, 491

Spruit, H. C. 1981, A\&SA, 98, 155

Spruit, H. C. 1982, Solar Phys., 75, 3 